\title{
Operative Versorgungskonzepte bei proximalen Femurfrakturen
}

\author{
Uwe Schweigkofler, Günter Gundlach, Reinhard Hoffmann
}

\section{Zusammenfassung}

Proximale Femurfrakturen gehören zu den häufigsten Frakturen des alten Menschen. Die demografische Entwicklung lässt eine weitere deutliche Zunahme dieser Verletzung erwarten. Eine rasche Mobilisation des operativ versorgten Menschen ist zur Vermeidung von Sekundärkomplikationen wie z.B. Thrombose, Pneumonie oder Dekubitus notwendig. Das erzielte Operationsergebnis und das verwendete Implantat sollte die sofortige postoperative Belastungsstabilität gewährleisten, da eine Teilbelastung oder Entlastung von den älteren Patienten nicht erwartet werden kann. Eine präoperative Frakturanalyse und
Planung erlaubt die Entscheidung zwischen einem extramedullären Osteosyntheseverfahren (z.B. DHS) oder den intramedullären Verfahren (z.B. PFN). Intramedulläre Osteosyntheseverfahren bieten eine höhere Primärstabilität und scheinen bei instabilen Frakturtypen das Implantat der Wahl zu sein. Intra- und postoperative Komplikationsmöglichkeiten müssen jedoch bedacht werden, um sie vermeiden bzw. adäquat therapieren zu können. Extramedulläre Verfahren haben nicht nur wegen des günstigeren Preises, sondern auch wegen ihrer sicheren Anwendbarkeit, vor allem bei den „stabilen“ Frakturen, nach wie vor ihren Stellenwert.

\section{Einleitung}

Neben der sog. distalen Radiusfraktur loco typico stellen die proximalen Femurfrakturen die typischen Verletzungen des älteren Menschen dar. Ursächlich hierfür sind nicht nur die oft vorliegende eingeschränkte Mobilität (Gangunsicherheit) und Fallneigung aus internistischer Ursache (Synkope), sondern vor allem auch die durch eine stärker ausgeprägte Osteoporose „geschwächten“ Knochen [1].

Demografische Untersuchungen zeigen den zunehmend hohen Anteil älterer Menschen an der Bevölkerung. Im Jahre 2002 wurden über 250000 Menschen als Folge eines Sturzereignisses stationär eingewiesen und es verstarben 12/ 100000 Einwohner (2001) an den Folgen eines Sturzes [2]. Für die prox. Femurfrak-

OP-JOURNAL 2005; 21: 128-132

(c) Georg Thieme Verlag KG Stuttgart · New York turen wird eine Inzidenz von 660/ 100000 in der Altersgruppe über 65 Jahre angegeben, bei Bewohnern von Altenund Pflegeheimen nimmt man eine Häufigkeit von 4000/100000 an [3].

Es muss zwischen den intraartikulär liegenden Schenkelhalsfrakturen (med. SHF), den extraartikulären lateralen Schenkelhalsfrakturen (lat. SHF) und den trochantären und subtrochantären proximalen Femurfrakturen unterschieden werden. Das Verhältnis zwischen Schenkelhalsfrakturen und trochantären Frakturen wird mit 1:1 angenommen.

Gerade aufgrund der häufig vorliegenden internistischen Begleiterkrankungen ist eine rasche operative Versorgung dieser Patienten mit dem Ziel einer frühen Mobilisierung angezeigt. Da die Patienten mit schon eingeschränkter Mobilität keine postoperative Entlastung oder Teilbelastung durchführen können, ergibt sich die Notwendigkeit einer stabilen Osteosynthese mit sofortiger postoperativer Belastungsfähigkeit.
Grundsätzlich stehen für die Versorgung von proximalen Femurfrakturen zwei klassische Versorgungssysteme zur Verfügung:

- das extramedullär stabilisierende Plattensystem der Dynamischen Hüftschraube (DHS)

- die intramedullär stabilisierenden Nagelsysteme (z.B. PFN,TFN oder PFN-A).

Die Versorgung mit Endernägeln oder Winkelplatten ist nicht mehr zeitgemäß und allenfalls noch Ausnahmeindikationen vorbehalten. Ebenso ist die Versorgung von prox. Femurfakturen mit Endoprothesen (z.B. Langschaftprothesen) derzeit noch Einzelfällen und Revisionseingriffen nach Implantatversagen vorbehalten.

Alte Menschen erfordern eine belastungsstabile Osteosynthese.

\section{Äthiologie und Klassifikation}

Durch den Sturz auf die Seite kommt es oft zu einem Varusstress in der Trochanterregion. Daraus resultieren überwiegend instabile Frakturen. Aufgrund der Ansätze großer Muskelgruppen (Glutealmuskulatur) und der etwas innerhalb der Trochanterregion verlaufenden Tragachse des Körpers liegen erhebliche Dislokationskräfte vor, die zudem noch auf kurze Bruchenden einwirken und die Stabilisierung erschweren. Bereits beim sitzenden Patienten wird die Trochanterregion mit $40 \%$ des Körpergewichts, bei Vollbelastung mit 280\% des Körpergewichts belastet [4].

Proximale Femurfrakturen werden üblicherweise anhand der AO-Klassifikation (Abb.1) [5] eingeteilt, wobei der Verlauf der Hauptbruchlinie, die Intaktheit der lat. Kortikalis und die med. Abstützung (Trochanter minor) entscheidende Kriterien darstellen.

A1-Frakturen: Verlauf von proximal-lateral nach distal-medial mit unverletzter 


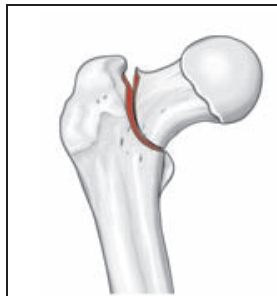

A1.1

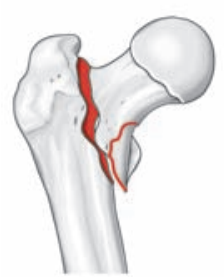

A2.1

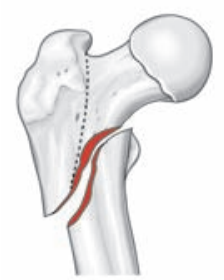

A3.1

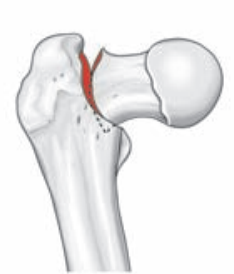

A1.2

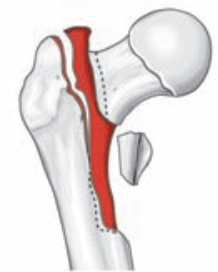

A2.2

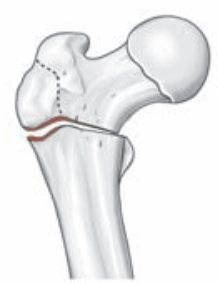

A3.2

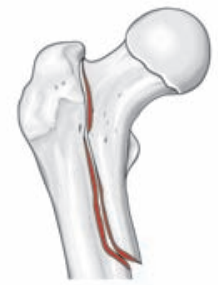

A1.3

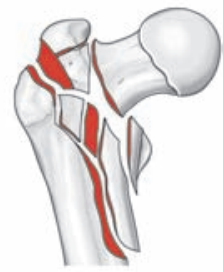

A2.3

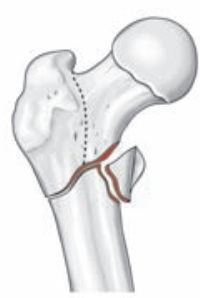

A3.3
Abb. 1 AO-Klassifikation der prox. Femurfrakturen. lat. Kortikalis und intaktem Trochanter major und minor.

(Beispiel: einfache pertrochantäre $\mathrm{Fe}$ murfraktur.)

A2-Frakturen: Pertrochantärer Frakturverlauf mit Beteiligung und ggf. Dislokation des Trochanter minor, woraus eine fehlende mediale Abstützung resultiert.

A3-Frakturen: Neben der Beteiligung des Trochanter minor ist durch den Frakturverlauf auch die lat. Kortikalis und der Trochanter major betroffen. Ein Sonderfall ist die sog. reversed fracture (A3.1) mit dem Verlauf von lateral-distal nach medial-proximal. Der Übergang zu den subtrochantären Frakturen oder Kombinationsfrakturen (per/subtrochantär), die durch Frakturausläufer bis unterhalb des Trochantor minors gekennzeichnet sind, ist oft fließend.

Die Versorgung von instabilen proximalen Frakturen stellen hohe Ansprüche an den Operateur und die Implantate.

\section{Klinilk und Diagnostik}

Die Patienten erlauben oft schon durch einen einfachen Blick auf die Beine eine Verdachtsdiagnose: aufgrund des Mus- kelzugs der Mm. glutei und der Außenrotatoren ist das verletzte Bein häufig verkürzt und außenrotiert. Die aktive und passive Beweglichkeit in der Hüftgelenksregion ist schmerzbedingt aufgehoben. Eine radiologische Abklärung ist unerlässlich. Diese besteht aus einer tief eingestellten Beckenübersichtsaufnahme sowie der Darstellung der betroffenen proximalen Femurregion a.p. und in der axialen Aufnahme. Vor allem die axiale Aufnahme ergibt Aufschlüsse über die Dislokation der Trochanteren bzw. des Schaftes und über die Schaftkrümmung, was für die Planung der operativen Versorgung (Implantatwahl, Lagerung, etc.) notwendig ist.

Aufgrund der meist vorliegenden Komorbidität und des Alters der Patienten gehören eine Thoraxübersichtsaufnahme, ein EKG sowie die Routinelabordiagnostik zur Standardvorbereitung.

Die Patienten sollten so schnell wie möglich operativ versorgt werden, um die aus der Immobilisation resultierenden Sekundärprobleme wie z.B. Thrombose oder Pneumonie zu vermeiden. Das anzustrebende Versorgungsfenster von 24 Stunden erlaubt aber in aller Regel die adäquate internistisch/anästhesiologische Op-Vorbereitung mit Einstellung des
Elektrolyt- und Wasserhaushaltes und Stabilisierung der kardio-pulmonalen Ausgangssituation. Untersuchungen zeigen, dass sowohl eine unzureichende OP-Vorbereitung als auch eine präoperative Vorbereitungsphase über $48 \mathrm{~h}$ die Rate der postoperativen Komplikationen deutlich erhöht [6]. Eine supracondyläre Extensionsbehandlung ist in der Regel nicht erforderlich da eine schmerzfrei Lagerung meist mit Lagerungshilfen (Kissen, Sandsack) gewährleistet werden kann.

Dem hohen Thromboserisiko der hüftgelenksnahen Verletzungen ist durch ein entsprechendes Therapieregime mit z.B. niedermolekularen Heparinen Rechnung zu tragen.

Gerade bei geriatrischen Patienten mit Desorientiertheit, Verwirrtheitszuständen oder einem Hirnorganischen Psychosyndrom (HOPS) muss unter Umständen beim Amtsgericht eine vorübergehende Betreuung nach §1896BGB eingeleitet werden.

\section{Präoperative Planung und Implantatwahl}

Entscheidend für die Auswahl des Implantates ist die Einschätzung der „Stabilität" des Bruches nach den präoperativen Bildern. Die zur Verfügung stehenden Implantate sind vielfältig und ihre Anwendung hängt, neben rein medizinischen Gründen, auch von ökonomischen und infrastrukturellen Überlegungen in den jeweiligen Versorgungseinrichtungen $a b$.

Wie oben ausgeführt, ist die Wiederherstellung der Belastbarkeit und damit eine Frühmobilisation oberstes Therapieziel. Die Implantate müssen den reponierten Knochen auch bei schlechter Knochenqualität und eingeschränktem Verankerungsraum sicher retinieren. Die Position der Trochanteren, die korrekte Achsstellung, ein annähernd normaler CCD-Winkel (Caput-Collum-Diaphysenwinkel ca. $130^{\circ}$ ) und ein guter Fragmentkontakt sind Voraussetzung für eine knöcherne Heilung.

Ein Ineinandergleiten der hüftgelenksnahen Anteile der Implantate durch das Gleitschraubenprinzip ermöglicht eine gute Frakturheilung unter Sinterung der Fraktur (dynamische Osteosynthese), worin sich neben der besseren Implantatstabilität die wesentlichen Vorteile zur Winkelplattenosteosynthese finden. Allen modernen Implantaten für den pro- 


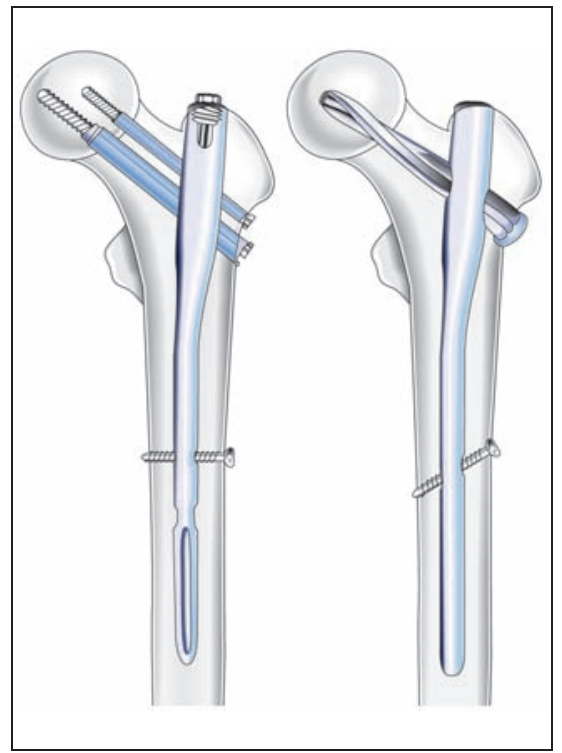

Abb. 2 PFN und PFN-A im Schema.

ximalen Oberschenkel ist die Krafteinleitung und Implantatfixierung im Hüftkopf und Schenkelhals gemeinsam.

- extramedulläre Systeme:

DHS (dynamische Hüftschraube)

- intramedulläre Systeme:

PFN (proximaler Femurnagel)

PFN-A

TFN (Trochanter Fixationsnagel)

Sowohl die intramedullären Systeme als auch die extramedullären Systeme bieten variable CCD-Winkel (z.B. PFN-A: 125$135^{\circ}$; DHS $135^{\circ}-150^{\circ}$ ) an.

Die Schenkelhalskomponente hat in der Regel eine mind. $10 \mathrm{~mm}$ starke Schenkelhalsschraube als Hauptlastträger, die z.B. beim PFN mit einer Antirotationsschraube fest kombiniert wird. Bei der DHS kann eine Antirotationsschraube optional in Freihandtechnik parallel zur Schenkelhalsschraube eingebracht werden.

Beim PFN-A und TFN ist die Rotationsund Winkelstabilität im Gegensatz zum PFN mit einem einzigen Element realisiert. Diese Klinge verdichtet beim Einschlagen die Spongiosa und ergibt auch beim osteoporotischen Knochen einen guten Halt und eine geringere Gefahr des Cut-out (Abb.2).

Die Krafteinleitung bzw. Implantatfixierung im Femurschaft erfolgt bei den extramedullären Systemen (DHS) duch die Fixation einer Platte mit Kortikalisschrauben an den Knochen. Die Plattenlänge kann entsprechend den Erfordernissen der Fraktur gewählt werden.
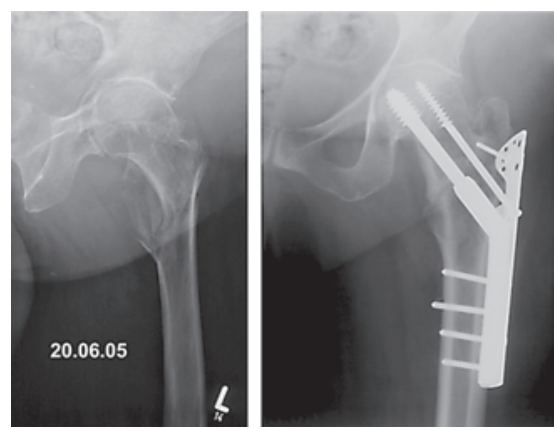

Abb.3 A2.3 Fraktur, Versorgung mit DHS mit Trochanterabstützplatte.

Durch die aufsteckbare Trochanterabstützplatte nach Regazzoni können auch instabile Frakturen mit Beteiligung des Trochanter major sicher versorgt werden (Abb.3). Die Abstützplatte fixiert den Trochanter major und verhindert eine Dislokation nach lateral, weiterhin können Trochanterfragmente gezielt mit Kleinfragmentschrauben oder additiver Nahtfixation an der Platte fixiert werden [7].

Bei den intramedullär stabilisierenden Systemen (z. B. TFN, Abb.4) wird das Implantat in der Markhöhle des Femurschaftes platziert, wodurch eine Weichteiltraumatisierung oder Durchblutungsstörungen durch den Zugang und das Implantat (Drucknekrosen des Periostes) deutlich reduziert werden. Auch bei den intramedullären Systemen stehen verschiedene Nagellängen und Stärken zur Auswahl. Distal erfolgt die Verriegelung des Implantates in der Regel statisch, wodurch die rasche Belastbarkeit gegeben ist.

In den meisten Fällen lassen sich die Frakturen alternativ mit den intra- oder extramedullär stabilisierenden Systemen ver- sorgen. Die Implantatwahl ist v.a. abhängig von der Infrastruktur des Krankenhauses und der Erfahrung des Operateurs mit dem Implantathandling. Rein wirtschaftlich betrachtet ist die DHS preisgünstiger als die intramedullären Systeme, in Kombination mit der Trochanterabstützplatte kommt sie jedoch auch wieder in diesen Bereich.

Bei A1-Frakturen mit intakter medialer Abstützung (d.h. erhaltenen Trochanter minor) ist die Versorgung mit einer DHS prinzipiell ausreichend.

Bei höhergradig instabilen Frakturen (A2 und A3) bieten die intramedulläre Systeme (PFN, PFN-A und TFN) eine etwas höhere Biegefestigkeit und Stabilität [8]. Bei den A3-Frakturen werden sie daher bevorzugt.

Bei komplexen Frakturen müssen gegebenenfalls zusätzlich Cerclagen zur Stabilisierung/Adaptation von langen subtrochantären Spiralfragmenten eingebracht werden. In solchen Fällen kann eine offene Reposition trotz der Verwendung des intramedullären Systemes notwendig werden.

Stark dislozierte Trochanter major Fragmente sollten refixiert werden. Bei der DHS mit der Trochanterabstützplatte oder durch Cerclagerefixation an den Schrauben der intramedullären Systeme.

Bei der geschlossenen Reposition dürfen keine Kompromisse eingegangen werden, im Zweifelsfall ist eine offene Reposition vorzunehmen.

Die korrekte Lage der Ziel K-Drähte im Schenkelhals ist in beiden Ebenen genauestens zu überprüfen und ggf. zu optimieren.
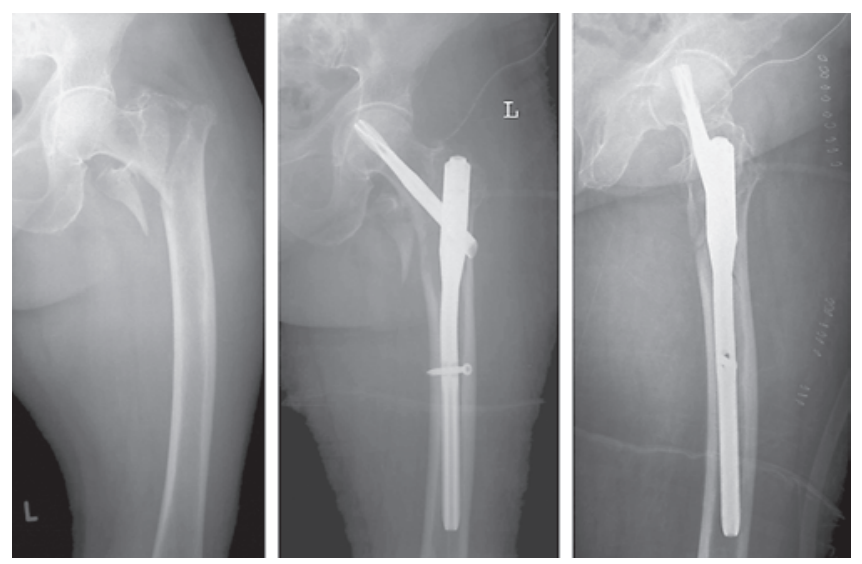

Abb. 4 A2.2 Fraktur, Versorgung mit TFN. 


\section{Operatives Vorgehen}

Die operative Versorgung proximaler Femurfrakturen erfolgt nach perioperativer single shot Antibiose in Rückenlage. Die Versorgung kann, zumindest bei den A1-Frakturen auf dem Standardtisch erfolgen. Wir bevorzugen jedoch den Extensionstisch (Abb.5), da dieses den intraoperativen Einsatz des Bildverstärkers erleichtert. Auf dem Extensionstisch wird noch unsteril eine geschlossene Reposition (meist durch ein Extensions-Innenrotations-Adduktionsmanöver) vorgenommen und die Brucheinrichtung in beiden Röntgenstandardebenen kontrolliert. Bei komplexen Verletzungen zeigt vor allem die axiale Einstellung noch bestehende Fehlstellungen (Absacken des Schaftfragmentes), die intraoperativ gegebenenfalls auch offen reponiert werden müssen.

Nach sterilem Abwaschen kann das Operationsgebiet mit der durchsichtigen Vertikalfolie abgeklebt werden. Alternative Abdeckungen kommen unter Umständen für die offene Reposition zum Einsatz.

Operativer Zugang für die Versorgung mittels:

- DHS: erfolgt als Längsschnitt (ca. 10$15 \mathrm{~cm}$ ) unterhalb des Trochanters major, Längsspaltung der Fascie des M. tensor latae und abpräparieren des M. vastus lateralis am Septum intermuskulare nach dorsal zur Darstellung des Femurs.

- Bei intramedullären Systemen: wird ein ca. $5 \mathrm{~cm}$ langer Hautschnitt oberhalb des Trochanters major angelegt und nach Faszienspaltung bis auf die Spitze des Trochanter major eingegangen. Die Kortikalis wird mit einem Steinmannnagel eröffnet und bei korrekter Lage (in beiden Ebenen in Verlängerung der Markhöhle) bis auf Höhe des Trochanter minor überbohrt. Der an den Zielbügel vormontierte Nagel wird intramedullär eingeführt.

Bei beiden Verfahren kommt der Platzierung der Zieldrähte für die Schenkelhalsschraube eine Schlüsselrolle zu. Um einen optimalen Halt im Schenkelhals/Hüftkopf zu ermöglichen, soll der Draht und somit später auch die Schraube in der a.p.-Projektion knapp oberhalb des Adamschen Bogens und in der axialen Ebene eher etwas dorsal des Hüftkopfzentrums zu liegen kommen. Bei den neueren Systemen wird eine zentrale Lage im Hüftkopf empfohlen. Es müssen Gewinde K-Drähte zur Vermeidung von Dislokationen verwendet werden. Der Abstand der Schenkel-

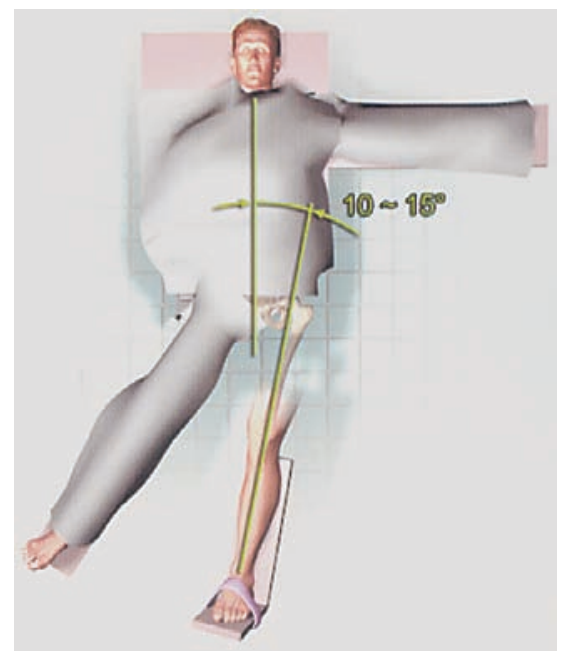

Abb. 5 Lagerung auf dem Extensionstisch (Schema).
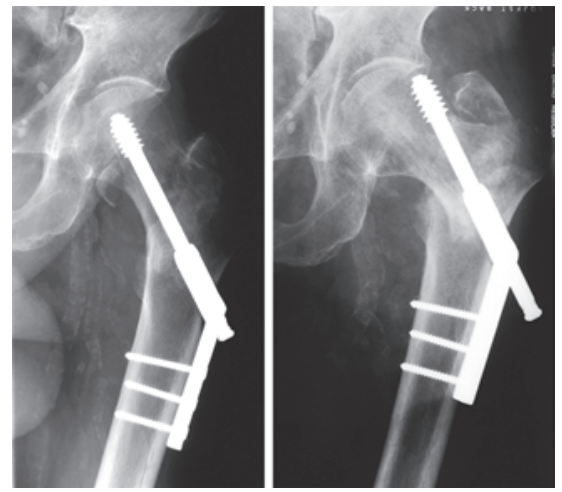

Abb. 6 DHS-Versorgung, Cut-out der Schraube.

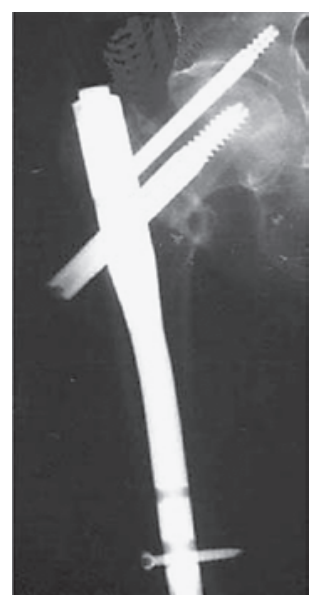

Abb. 7 Z-Phänomen bei PFNVersorgung.

halsschraube von der Kopfzirkumferenz sollte $1-1,5 \mathrm{~cm}$ betragen.

Nach Positionierung der Schenkelhalsschraube (und einer Antirotationsschraube, fakulativ bei der DHS) wird bei der DHS die Laschenplatte aufgeschoben und am Schaft mit Kortikalisschrauben fi- xiert. Beim PFN wird der Nagel über das Zielinstrumentarium im Schaft verriegelt.

\section{Intraoperative und postoperative Komplikationsmöglichkeiten}

Eine ungenügende bzw. eine fehlerhafte Reposition kann naturgemäß durch kein Implantat kompensiert werden, woraus sich die besondere Bedeutung der geschlossenen Reposition und der prä-/intraoperativen BV-Kontrollen erklärt. Bei schwierigen Repositionsmanövern kann ein korrekturbedürftiger Innenrotationsfehler resultieren.

In der Literatur wird die Rate der Gesamtkomplikationen bei der Versorgung von prox. Femurfrakturen mit $14 \%$ angegeben [10].

Folgende typische Komplikationen sind für die intra- und extramedullären Systeme zu nennen:

- DHS:

- Korrekturverlust

- Cut-out

- Sinterung

- Lateralisation des Schenkelhalses/Trochantermassivs

Die Schenkelhalsschraube betreffend, kann es bei beiden Systemen neben einer initialen Fehllage zu einem sog. Cut-out kommen. D.h. die Schenkelhalskomponente schneidet durch die weiche Kopfspongiosa und bricht schließlich in das Hüftgelenk aus (Abb.6). Für die DHS ist dieses Problem in einer AO-Sammelstatistik mit 2,6\% beschrieben [9].

Bei der DHS besteht die Gefahr der Frakturierung der lateralen Kortikalis im Bereich der Aufbohrung (3.Stufe des Stufenbohrers).

Bei der DHS ist als Implantatversagen das Ausreißen der Platte aus dem Schaft zu nennen.

- PFN:

- Cut-out

- Z-Effekt

- Implantatdislokation/bruch

- Schaftperforation/fraktur bei starker Femurschaftantekurvation

Bei osteosynthetischen Versorgungen mit dem PFN können Schenkelhalsschraubendislokation beobachtet werden. Eine zentrale Dislokation ins Becken ist durch ein verändertes Design am Schraubenende verhindert worden. Das sog. Z-Phänomen (Abb. 7), d.h. das Zusammensintern der Fraktur mit Auswandern der Schen- 
kelhalsschraube und Perforation der Kopfkalotte durch die nicht auswandernde Antirotationsschraube [13] wird beim PFN noch beobachtet. Der TFN und PFN-A trägt diesem Problem durch die Umstellung auf das Klingensystem Rechnung. Bei starker Fraktursinterung und Auslockern der Schenkelhalsschraube kann als „kleiner“ Revisionseingriff mit einem Schraubentausch nach Auffüllen des Bohrkanals mit Knochenzement versucht werden, die endoprothetische Versorgung zu vermeiden. Beim Überbohren des Nageleintrittes im Trochanterbereich, kann es v.a. bei Frakturen in diesem Bereich zu einer erheblichen Fragmentdislokation kommen.

Die durch die intramedullären Systeme provozierte Schaftfraktur im Bereich des distalen Nagelendes wird beim PFN in $3 \%$ beschrieben. Beim $\gamma$-Nagel wurde diese Komplikation wegen der relativen Stärke und Rigidität des Implantates deutlich häufiger (bis 12\%) beobachtet. Ursächlich ist die fehlende Antekurvation des kurzen Nagels, wodurch er bei starker Krümmung des Femurschaftes zu Fissuren des Schaftes im Nagelspitzenbereich führen kann. So etwas passiert vor allem beim Einschlagen des Nagels mit dem Hammer um die Schenkelhalsschraube unmittelbar über den Adamschen Bogen zu platzieren [11]. Zur Vermeidung dieser Komplikation ist bei starker Antekurvation des Femurschaftes die Versorgung mit dem langen entsprechend vorgebogenen Nagel zu nennen, auch wenn die eigentliche Fraktur kein langes Implantat erfordern würde.

Als weitere Komplikation ist das eigentliche Implantatversagen, d.h. der Implantatbruch zu beschreiben.

Bei beiden Versorgungssystemen (intramedullär/extramedullär) sind Serome, Hämatome und tiefe Infekte als Komplikationen zu nennen, die bei den extramedullären Verfahren treten diese Komplikationen aufgrund der Zugangsmorbidität etwas häufiger auf.

Die Hüftkopfnekrose stellt bei den extrakapsulären trochantären Frakturen eine Ausnahme dar.

Die Gesamtletalität dieser Frakturen wird mit $4 \%$ angegeben [12].
Revisionsoperationen nach der Versorgung von proximalen Femurfrakturen infolge von Implantatversagen oder Komplikationen werden in einer Häufigkeit von $2-12 \%$ angegeben.

Das Einschlagen des PFN mit dem Hammer kann, bei starker Krümmung des Femurschaftes, Frakturen an der Implantatspitze provozieren und ist unter Umständen durch den Wechsel auf einen langen PFN mit entsprechender Antekurvation zu vermeiden.

\section{Fazit}

Die zur Verfügung stehenden extramedullären (DHS) oder intramedullären (PFN, PFN-A, TFN) Implantate ermöglichen in einem hohen Prozentsatz die belastungsstabile osteosynthetische Versorgung von proximalen Femurfrakturen des alten Menschen. Bei der operativen Versorgung muss den häufig vorliegenden Begleiterkrankungen (z.B. KHK, Demenz, pAVK) ebenso Rechnung getragen werden, wie der meist schlechten Knochenqualität. Revisionseingriffe nach fehlgeschlagenen Osteosynthesen sind für den Patienten mit einem sehr hohen Risiko verbunden. Eine adäquate kardiopulmonale Vorbereitung innerhalb von $24 \mathrm{~h}$ ist für das postoperative Ergebnis ebenso vorteilhaft wie die differenzierte Implantatwahl. Die beschriebenen intra- und postoperativen Komplikationen lassen sich durch entsprechende Vorsicht oft vermeiden. Die Versorgung auf dem Extensionstisch stellt sich hilfreich dar. Beim Repositionsergebnis dürfen keine Zugeständnisse gemacht werden, im Zweifel ist eine offene Reposition und die additive Verwendung von Cerclagen in der subtrochantären Region notwendig. Durch die so erzielte operative Versorgung können die älteren Patienten so rasch als möglich mobilisiert und in ihre gewohnte Umgebung entlassen werden.

\section{Literatur}

${ }^{1}$ Ford CM, Keaveny TM, Hayes WC. The effect of impact direction on the structural capacity of the proximal femur during falls. J Bone Miner Res 1996; 1: 377-383

${ }^{2}$ Statistisches Bundesamt (StBA) Wiesbaden (2002) Statistisches Jahrbuch 2002 (Reihe 7)

${ }^{3}$ Becker C, Scheible S. Stürze und sturzbedingte Verletzungen älterer Menschen. Fortschr. Med 1998; 116/32: 22-29
${ }^{4}$ Bergmann G, Rohlmann A, Graichen F. In vivo Messungen der Hüftgelenkbelastung 1.Teil: Krankengymnastik. Z. Orthop 1989; 127: $672-679$

${ }^{5}$ Müller ME, Allgöwer M, Schneider R, Willenegger H. Manual of internal fixation. Springer, Berlin, Heidelberg, New York 1991

6 Parker MJ, Pryor GA. The timing of the surgery for proximal femural fraktures. J Bone Joint Surg Br 1992; 74: 203-264

${ }^{7}$ Dávid A, Hufner T, Lewandrowski K, Papa D, Muhr $G$. The dynamic hip screw with support plate - a reliable osteosynthesis for highly unstable „reverse“ trochanteric fractures? Chirurg 1996; 67: 1166-1173

${ }^{8}$ Curtis MJ, Jinnah RH, Wilson V, Cunningham BW. Proximal femoral fraktures, a biomechanical study to compare intramedullary and extramedullary fixation. Injury 1994; 25: $99-104$

${ }^{9}$ Stürmer KM, Dreising K. Pertrochantäre Frakturen. Zbl Chir 1995; 120: 862-87

${ }^{10}$ Josten Ch, Tiemann AH. Komplikationen nach proximalen Femurfrakturen. OP-Journal 2002; 17: $148-153$

${ }^{11}$ Werner-Tutschku W, Lajtai G, Schmiedhuber $\mathrm{G}$, Orthner E. Intra- und perioperative Komplikationen von per- und subtrochantären Femurfrakturen mittels PFN. Der Unfallchirurg 2002; 105: $881-885$

${ }^{12}$ Zuckermann J, Skovron ML, Koval K, Aharonoff G, Frankel V. Postoperative complications and mortality associated with operative delay in older Patients who have a fracture of the hip. JBJS AM 1995; Vol 77-A(10): $1551-1556$

${ }^{13}$ Weise K, Schwab E. Intrameduläre kraftträger zur Versorgung der per- und subtrochantären Femurfraktur. Chirurg 2001; 72 : $1277-1282$

\section{Dr. med. Uwe Schweigkofler}

Oberarzt

BG-Unfallklinik Frankfurt

Friedberger Landstr. 430

D-60389 Frankfurt

\section{Dr. med. Gunter Gundlach}

Oberarzt

Klinikum Offenbach $\mathrm{GmbH}$

Starkenburgring 66

D-63069 Offenbach

Prof. Dr. med. Reinhard Hoffmann

Ärztlicher Direktor und Chefarzt der Abteilung Unfallchirurgie

BG-Unfallklinik Frankfurt

Friedberger Landstr. 430

D-60389 Frankfurt

Chefarzt Chirurgie II

Klinikum Offenbach $\mathrm{GmbH}$

Starkenburgring 66

D-63069 Offenbach 